\title{
Mucosal melanoma of the head and neck
}

\author{
SAYAKA KANETAKA, MAMORU TSUKUDA, MASAHIRO TAKAHASHI, \\ MASANORI KOMATSU, TATSUO NIHO, CHOICHI HORIUCHI and HIDEKI MATSUDA
}

\author{
Department of Otolaryngology, Head and Neck Surgery, \\ Yokohama City University School of Medicine, Kanagawa 236-0004, Japan
}

Received May 5, 2011; Accepted June 28, 2011

DOI: $10.3892 / \mathrm{etm} .2011 .313$

\begin{abstract}
Mucosal melanoma of the head and neck (MMHN) is a rare malignant tumor associated with a poor prognosis. A retrospective study of case records of patients treated at our department between 1992 and 2010 was carried out. Thirteen patients were enrolled. The median age of the patients (3 males and 10 females) was 61 years (range 39-78). The median follow-up period was 48 months (range 10-115). Two common primary sites were the nasal cavity ( 8 cases) and sinonasal complex (5 cases). Ten patients (77\%) received curative surgery. Chemotherapy was administered to 10 patients. In addition, lymphokine-activated killer (LAK) cell therapy was administered to 7 patients as adjunctive immunotherapy after the initial treatment course. The overall 5-year, cause-specific survival rate was $56 \%$. Patients who received adjunctive LAK cell therapy had a survival rate of $67 \%$ at 5 years, while patients who did not receive adjunctive LAK cell treatment had a survival rate of $33 \%$. MMHN is associated with a poor survival rate. The most common cause of death is distant metastasis. Surgery, radiotherapy and chemotherapy are common strategies for MMHN, but the control of metastasis is difficult. The use of immunotherapy remains uncommon for MMHN. However, from the viewpoint of a systemic disease, due to its high rate of metastases, immunotherapy using LAK cell treatment may contribute to the improvement of prognosis in patients with MMHN.
\end{abstract}

\section{Introduction}

Head and neck mucosal melanoma (HNMM) is a rare malignant tumor worldwide, but is relatively common in Japan,

Correspondence to: Dr Mamoru Tsukuda, Department of Otolaryngology, Head and Neck Surgery, Yokohama City University School of Medicine, 3-9 Fukuura Kanazawa-ku, Yokohama, Kanagawa 236-0004, Japan

E-mail:mtsukuda@med.yokohama-cu.ac.jp

Abbreviations: MMHN, mucosal melanoma of the head and neck; LAK, lymphokine-activated killer

Key words: mucosal melanoma of the head and neck, immunotherapy, lymphokine-activated killer cells comprising $6 \%$ of all melanomas (1). Resection with a clear margin is emphasized as the most important factor for good local control and better prognosis. However, radical surgery is not possible in certain cases as many important organs may be located near the tumor. In addition, effective chemotherapy has not yet been established, and a common therapeutic strategy has not been clarified excluding curative surgical resection.

This study was performed to analyze 13 cases of MMHN treated at the Yokohama City University School of Medicine from June 1992 to November 2010.

\section{Patients and methods}

The medical records of 13 MMHN patients treated at the Yokohama City University School of Medicine from June 1992 to November 2010 were retrospectively reviewed. Collected data included gender, age, primary site, extent of the lesion, nodal involvement at presentation, metastasis at presentation, treatment contents, positive or negative surgical margins and treatment outcome.

Patients consisted of 3 males and 10 females. Median age was 60.8 years (range 39-78). Common symptoms were epistaxes (38.5\%) and nasal obstruction (30.8\%). In terms of the primary site, tumors in 5 cases were in the paranasal cavities (maxillary sinus in 2 cases, ethmoid sinus in 2 cases, sphenoid sinus in 1 case) and 8 cases in the nasal cavity. Staging according to the Ballantyne's staging system was applied (1). Stage I: 11 cases showed lesional type tumors. Stage II: 1 case had cervical nodal metastasis only. Stage III: 1 case had distant metastases. Furthermore, Stage I was classified into two types. Tumors in 5 cases in Stage Ia were limited to one anatomical lesion, and those in 6 cases in Stage Ib extended to the adjacent lesion.

The median follow-up period was 48 months (range 10-115). Survival rates were expressed using the KaplanMeyer method and the Wilcoxon test was used to evaluate the difference between each group (Table I).

Surgical resection. Ten out of 13 patients underwent definitive surgery as initial treatment. Three patients did not undergo surgical operation due to 1 case each of liver metastases, orbital and skull base invasion, and skull base invasion.

Chemotherapy and radiotherapy. In addition to surgery, chemotherapy and/or immunotherapy were carried out in the 
Table I. Characteristics of 13 patients with mucosal melanoma of the head and neck.

\begin{tabular}{lc}
\hline Characteristics & No. \\
\hline Age (years) & 39-78 (average 61) \\
Gender & 3 \\
Male & 10 \\
Female & \\
Sites & 8 \\
Nasal cavity & 5 \\
Sinonasal cavity & 2 \\
Maxillary sinus & 2 \\
Ethmoidal sinus & 1 \\
Sphenoidal sinus & \\
Tumor stage & 5 \\
Stage Ia & 6 \\
Stage Ib & 1 \\
Stage II & 1 \\
Stage III & \\
\hline
\end{tabular}

initial treatment course (Table II). Chemotherapy was based on the so-called DAV therapy: Dimethyltriazeno-imidazole carboxamide (DTIC; 80-140 mg/m²), amino methyl pyrimidinyl methyl chlorethyl nitrorosourea hydrochloride (ACNU; $50-80 \mathrm{mg} / \mathrm{m}^{2}$ ) and vincristine (VCR; 0.5-0.8 mg/m²). Cisplatin with DAV was administered for 3 patients and interferon- $\alpha$ with DAV was applied in 5 cases (Table III).

Radiotherapy during initial treatment was carried out in 2 cases. Postoperative radiotherapy (50 Gy) was carried out for 1 case and heavy particulate radiotherapy was carried out for 1 inoperative case.
Immunotherapy. As for immunotherapy, lymphokine-activated killer (LAK) cell transfer therapy $(2,3)$ was carried out for 7 patients as adjunctive treatment after initial treatment. LAK cell therapy populates the self-activated lymphocytes intravenously with interleukin-2 (IL-2). Grimm et al (4) and Rosenberg et al (5) demonstrated an increasing trend in the survival rate when IL-2 was administered with LAK cells in patients with advanced cancer. Blood $(40-50 \mathrm{ml})$ was collected from the patients. Approximately $10^{9}$ lymphocytes were prepared after culture with the anti-CD3 antibody and IL-2, and subsequently CD-8-positive killer (LAK) cells were induced and proliferated. The average number of adjunctive LAK cell treatments was 16 (range 6-32). Many patients received the therapy once a week or once every 2 weeks. The number of cells injected each time was $5 \times 10^{8}$ to $5 \times 10^{9}$.

\section{Results}

The overall 5-year, cause-specific survival rate was 56\% (Fig. 1). Each rate showed a better outcome than those of other reports $(6,7)$. As for the operative therapy, the 5-year diseasefree survival rate in margin-positive surgery $(n=3)$ was $0 \%$, whereas it was $50 \%$ in margin-negative surgery $(n=7)(p=0.21)$ (Fig. 2). In 7 cases receiving adjunctive LAK cell therapy, the 5-year cause-specific survival rate was $66 \%$, while that in 6 cases without adjunctive LAK therapy was $33 \%(\mathrm{p}=0.43)$ (Fig. 3). Although a statistical significance was not recognized, LAK therapy is suggested to improve prognosis of MMHN.

\section{Discussion}

The incidence of malignant melanoma in Japan is low compared to Western countries. However, regarding the incidence of MMHN, in Western countries it accounts for only $1.7 \%$ of all cases of melanoma, whereas it accounts for $23.3 \%$ in Japan

Table II. Characteristics and outcomes of each patient.

\begin{tabular}{|c|c|c|c|c|c|c|c|c|c|}
\hline $\begin{array}{l}\text { Pt. } \\
\text { no. }\end{array}$ & Age & Gender & $\begin{array}{c}\text { Major } \\
\text { complaint }\end{array}$ & Site & Stage & $\begin{array}{l}\text { Primary } \\
\text { treatment }\end{array}$ & $\begin{array}{l}\text { Surgical } \\
\text { margin }\end{array}$ & $\begin{array}{l}\text { Adjunct LAK } \\
\text { cell therapy } \\
\text { (no. of times) }\end{array}$ & $\begin{array}{c}\text { Outcome } \\
\text { (follow-up period, } \\
\text { months) }\end{array}$ \\
\hline 1 & 45 & $\mathrm{~F}$ & Cheek pain & MS & $\mathrm{Ib}$ & $\mathrm{C} \rightarrow \mathrm{O} \rightarrow \mathrm{R}$ & - & - & Death (13) \\
\hline 2 & 67 & M & Epistaxis & $\mathrm{N}$ & Ia & $\mathrm{C} \rightarrow \mathrm{O} \rightarrow \mathrm{I}$ & - & - & Death (38) \\
\hline 3 & 54 & M & Epistaxis & $\mathrm{ES}$ & Ia & $\mathrm{C} \rightarrow \mathrm{I}$ & & - & Death (35) \\
\hline 4 & 74 & $\mathrm{~F}$ & Nasal obstruction & $\mathrm{N}$ & Ia & $\mathrm{O} \rightarrow \mathrm{I}$ & + & $+(32)$ & Death (64) \\
\hline 5 & 54 & $\mathrm{~F}$ & Nasal obstruction & $\mathrm{N}$ & Ia & $\mathrm{O} \rightarrow \mathrm{I}$ & - & $+(6)$ & Death (42) \\
\hline 6 & 68 & $\mathrm{~F}$ & Nasal obstruction & $\mathrm{N}$ & Ia & $\mathrm{C} \rightarrow \mathrm{O} \rightarrow \mathrm{I}$ & - & $+(13)$ & Death (115) \\
\hline 7 & 40 & $\mathrm{~F}$ & Epistaxis & $\mathrm{N}$ & $\mathrm{Ib}$ & $\mathrm{C} \rightarrow \mathrm{O} \rightarrow \mathrm{I}$ & + & $+(15)$ & Death (35) \\
\hline 8 & 56 & M & Epistaxis & $\mathrm{N}$ & $\mathrm{Ib}$ & $\mathrm{O} \rightarrow \mathrm{I}$ & + & $+(23)$ & Death (89) \\
\hline 9 & 74 & $\mathrm{~F}$ & Nasal obstruction & $\mathrm{N}$ & $\mathrm{Ib}$ & $\mathrm{O} \rightarrow \mathrm{C} \rightarrow \mathrm{I}$ & - & - & Alive (65) \\
\hline 10 & 40 & $\mathrm{~F}$ & Asymptomatic & SS & III & $\mathrm{R} \rightarrow \mathrm{C} \rightarrow \mathrm{I}$ & & $+(22)$ & Alive (62) \\
\hline 11 & 72 & $\mathrm{~F}$ & Exophthalmos & MS & $\mathrm{Ib}$ & $\mathrm{C} \rightarrow \mathrm{I}$ & & - & Alive (31) \\
\hline 12 & 69 & $\mathrm{~F}$ & Neck tumor & MS & II & $\mathrm{C} \rightarrow \mathrm{O} \rightarrow \mathrm{I}$ & - & - & Alive (31) \\
\hline 13 & 78 & $\mathrm{~F}$ & Epistaxis & $\mathrm{N}$ & $\mathrm{Ib}$ & $\mathrm{C} \rightarrow \mathrm{O} \rightarrow \mathrm{I}$ & - & $+(6)$ & Alive (10) \\
\hline
\end{tabular}

MS, maxillary sinus; ES, ethmoidal sinus; SS, sphenoidal sinus; N, nasal cavity. C, chemotherapy; O, operation; I, immunotherapy. 
Table III. Chemotherapy regimens.

\begin{tabular}{ll}
\hline $\mathrm{DAV}+\mathrm{CDDP}$ & 3 \\
$\mathrm{DAV}$ & 2 \\
DAV-feron & 5
\end{tabular}

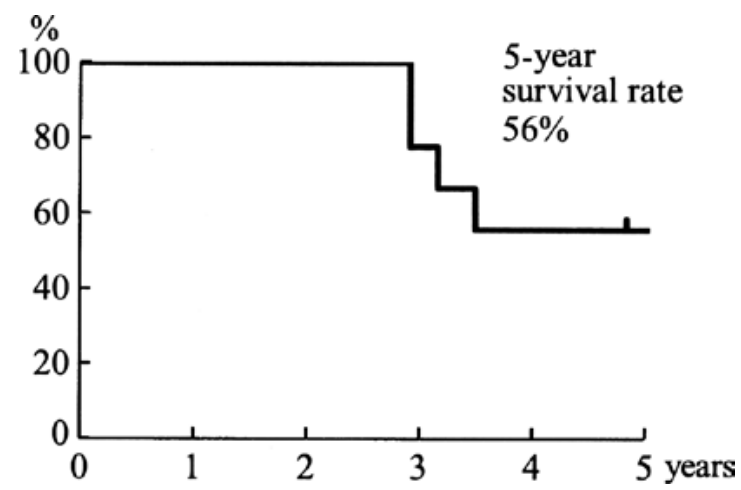

Figure 1 . The 5-year cause-specific survival rate was 56\%, which showed a better outcome than those of other reports.

$(8,10)$. MMHN arises from aberrant melanocytes during fetal life. On the other hand, a difference in sensitivity to ultraviolet rays is assumed to cause skin melanoma. Therefore, racial difference is considered to be associated with a high incidence of melanoma of the skin in Western countries.

With regard to gender and $\mathrm{MMHN}$, certain reports have shown a male-to-female ratio of $1: 1$ to $2: 1(8,10)$. In this study, the ratio was 3:10. Regarding the primary site of $\mathrm{MMHN}$, various studies have reported a rate of occurrence of $50 \%$ in the oral cavity and $35 \%$ in the sinonasal cavity (10), or a high occurrence in the sinonasal cavity (11). In this study, 8 cases $(61 \%)$ originated from the nasal cavity and 5 cases $(39 \%)$ from the paranasal cavities.

A treatment modality for MMHN has yet to be established. For resectable lesions, complete removal with surgical margins, such as a basilar operation, is generally the most optimal course. Regarding chemotherapy for MMHN, a DTIC-based combination regimen is often applied (12). Penel et al reported that positive surgical margins were a risk factor in univariate analysis, and they suggested that clear margins appeared to predict a more favorable outcome (13). Other reports also revealed that the quality of the margins was linked to a better overall survival rate $(10,14)$. Thus, operative therapy is the first choice for curative treatment. In our study, the 5-year diseasefree survival rate in margin-positive surgery $(n=3)$ was $0 \%$, whereas it was $50 \%$ in margin-negative surgery $(n=7)(p=0.21)$ (Fig. 2).

On the other hand, recent reports have shown almost the same effectiveness in terms of local control by high-dose fractionated radiotherapy in comparison to curative surgery $(7,15)$. Wada et al reported that high-dose per fractionated radiotherapy doses ( $\geq 3 \mathrm{~Gy}$ ) were associated with better prognosis for both local control and survival (9). The effect of postoperative radiotherapy has also been the focus of research (14). Temam et al demonstrated that patients with early T-classification tumors who received postoperative radio-

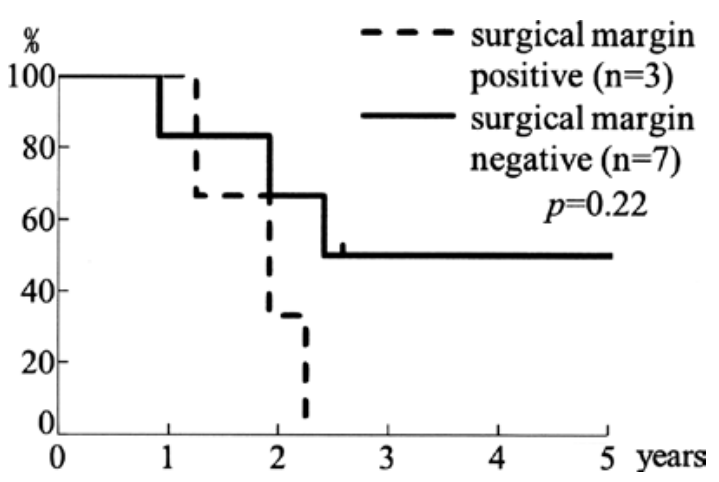

Figure 2. Disease-free survival rate of patients with positive/negative surgical margins. The 5-year disease-free survival rate in margin-positive surgery was $0 \%$, whereas it was $50 \%$ in margin-negative surgery, indicating that curative surgery contributes to better disease control.

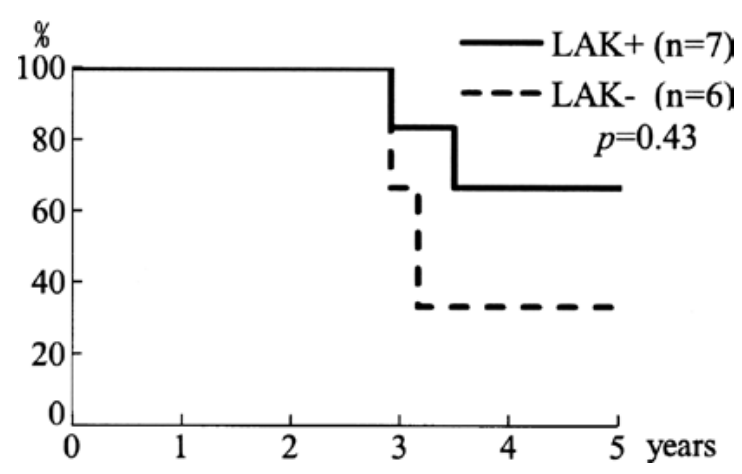

Figure 3. Cause-specific survival rate in patients with/without adjunctive LAK cell therapy. In cases receiving adjunctive LAK therapy, the 5-year cause-specific survival rate was $67 \%$, while in cases without adjunctive LAK therapy, it was $33 \%$.

therapy had a better local disease-free survival compared to patients with late T-classification tumors who did not receive postoperative radiotherapy (16). Thus, the role of radiotherapy, such as high-dose fractionated radiotherapy and carbon ion radiotherapy, is expected to be considerably more important for functionally and cosmetically operable cases $(9,17,18)$. However, radiotherapy treats the local tumor, but does not inhibit distant metastasis $(14,16)$. A combination treatment with systemic therapies, such as chemotherapy and immunotherapy, is indispensable for the improvement of the outcome of MMHN $(14,16)$.

As for immunotherapy, LAK cells were administered intravenously with IL-2 to retain its activity in attacking cancer cells. Grimm et al (4) and Rosenberg et al (5) found an increased trend in the survival rate when IL-2 was administered with LAK cells in patients with melanoma. Seven patients received LAK cell therapy as adjunctive therapy after initial treatment. The 5-year cause-specific survival rate of this group was $67 \%$, while that of 6 cases without LAK cell therapy was $33 \%(\mathrm{p}=0.43)$. Due to the small population, a statistical significance could not be obtained. However, based on the results, LAK cell therapy is suggested to improve prognosis of MMHN (Fig. 3).

Because of the small size of most reported series and their retrospective nature, the effect of various treatment strategies for MMHN has been difficult to evaluate. 
Surgical treatment has been the primary treatment modality. However, as wide surgical resection in the head and neck region is often difficult, radiotherapy has been recently reported as a beneficial management modality. The high rate of distant metastasis also suggests that a systemic treatment is required. Currently, there is no role for adjuvant systemic therapy for patients who have been successfully resected. Further evaluation of the role of chemotherapy and immunotherapy is required to decrease the rates of distant metastasis and improve survival (19).

There are few reports on immunotherapy for MMHN. Based on dermatological research, malignant melanoma is targeted using immunotherapies, such as cytokines, cancer vaccines, adoptive cell transfer and cancer gene therapies, and these immunotherapies have been actively investigated (20-22). For example, Atkins et al reported that 12 responding patients out of 270 patients with metastatic melanoma treated with high-dose recombinant IL-2 therapy remained continually disease-free or progression-free from $>70$ to $>150$ months following initiation of therapy (20).

In the present study, LAK cell therapy as initial or adjunctive therapy for patients with MMHN was applied. A tendency for an improved survival rate of patients with MMHN by LAK cell therapy was recognized. Further investigation using an accumulation of cases is warranted.

\section{References}

1. Ballantyne AJ: Malignant melanoma of the skin of the head and neck. An analysis of 405 cases. Am J Surg 120: 425-431, 1970

2. Tsukuda M, Mochimatsu I, Sakumoto M, et al: The synergistic effects of interleukin 2 and interleukin 7 on the proliferation and autologous tumor cell lysis of tumor-associated lymphocytes. Biotherapy 6: 167-174, 1993.

3. Tsukuda M, Mochimatsu I, Sakumoto M, et al: Autologous tumor cell killing activity of tumor-associated lymphocytes in patients with head and neck carcinomas. Biotherapy 6: 155-161, 1993

4. Grimm EA, Mazumder A, Zhang HZ, et al: Lymphokineactivated killer cell phenomenon. Lysis of natural killer-resistant fresh solid tumor cells by interleukin 2-activated autologous human peripheral blood lymphocytes. J Exp Med 155: 1823-1841, 1982.

5. Rosenberg SA, Lotze MT, Yang JC, et al: Prospective randomized trial of high-dose interleukin-2 alone or in conjunction with lymphokine-activated killer cells for the treatment of patients with advanced cancer. J Natl Cancer Inst 85: 622-632, 1993.
6. Manolidis S and Donald PJ: Malignant mucosal melanoma of the head and neck: review of the literature and report of 14 patients. Cancer 80: 1373-1386, 1997.

7. Douglas CM, Malik T, Swindell R, et al: Mucosal melanoma of the head and neck: radiotherapy or surgery? J Otolaryngol Head Neck Surg 39: 385-392, 2010.

8. Omura K, Takemiya S, Shimada F, et al: Malignant mucosal melanomas of the head and neck - collective review from six cancer hospitals. Gan No Rinsho 32: 1511-1518, 1986.

9. Wada H, Nemoto K, Ogawa Y, et al: A multi-institutional retrospective analysis of external radiotherapy for mucosal melanoma of the head and neck in Northern Japan. Int J Radiat Oncol Biol Phys 59: 495-500, 2004.

10. Conley JJ: Melanomas of the mucous membrane of the head and neck. Laryngoscope 99: 1248-1254, 1989.

11. Saito H, Hozawa J and Yamagami M: Statistical analysis of malignant melanoma of nasal cavity and paranasal sinuses in Japan. Nippon Jibiinkoka Gakkai Kaiho 86: 139-143, 1983.

12. Ishihara K, Yamazaki $\mathrm{N}$ and Asano K: Chemotherapy of malignant melanoma. Gan To Kagaku Ryoho 20: 1287-1292, 1993.

13. Penel N, Mallet Y, Mirabel X, et al: Primary mucosal melanoma of head and neck: prognostic value of clear margins. Laryngoscope 116: 993-995, 2006.

14. Moreno MA, Roberts DB, Kupferman ME, et al: Mucosal melanoma of the nose and paranasal sinuses, a contemporary experience from the M. D. Anderson Cancer Center. Cancer 116: 2215-2223, 2010.

15. Mizoe JE, Tsujii H, Kamada T, et al: Dose escalation study of carbon ion radiotherapy for locally advanced head-and-neck cancer. Int J Radiat Oncol Biol Phys 60: 358-364, 2004.

16. Temam S, Mamelle G, Marandas P, et al: Postoperative radiotherapy for primary mucosal melanoma of the head and neck. Cancer 103: 313-319, 2005.

17. Zenda S, Kawashima M, Nishio T, et al: Proton beam therapy as a nonsurgical approach to mucosal melanoma of the head and neck: a pilot study. Int J Radiat Oncol Biol Phys: Oct. 13. 2010 (E-pub ahead of print).

18. Trotti A and Peters LJ: Role of radiotherapy in the primary management of mucosal melanoma of the head and neck. Semin Surg Oncol 9: 246-250, 1993.

19. Gavriel H, McArthur G, Sizeland A and Henderson M: Review: mucosal melanoma of the head and neck. Melanoma Res: April 30, 2011 (E-pub ahead of print).

20. Atkins MB, Kunkel L, Sznol M, et al: High-dose recombinant interleukin-2 therapy in patients with metastatic melanoma: long-term survival update. Cancer J Sci Am 6 (Suppl 1): 11-14, 2000.

21. Baba T, Sato-Matsushita M, Kanamoto A, et al: Phase I clinical trial of the vaccination for the patients with metastatic melanoma using gp100-derived epitope peptide restricted to HLA-A*2402. J Transl Med 8: 84, 2010.

22. Rosenberg SA, Restifo NP, Yang JC, et al: Adoptive cell transfer: a clinical path to effective cancer immunotherapy. Nat Rev Cancer 8: 299-308, 2008. 